\title{
LA RAISON POLÉMIQUE CHEZ JOHN TOLAND Michel MalHERBE
}

RÉsuMÉ : L'idée d'une raison polémique est-elle viable ? On la met ici à l'épreuve en tentant de définir le style philosophique de John Toland, style qui est à la fois un mode de pensée et un mode d'écriture. On étudie ses conditions et ses formes. Il apparaît chez John Toland que cette raison polémique, qui prend en charge le combat contre l'intolérance et reste constamment attentive à la communication entre les esprits, ressortit plus à un genre de prédication qu'au raisonnement, et que ses fins sont principalement pratiques.

Mots clés : polémique, l'oral et l'écrit, l'art de la communication, autorité, évidence, raison.

Summary: Does the idea of a polemical reason make sense? This idea is here tested through an attempt to define John Toland's philosophical style, both as a way of thinking and $a$ way of writing. Its conditions and forms are studied. It appears that in Toland's works this polemical reason, which is the means of fighting intolerance and making easier a certain kind of philosophical communication, relies on preaching rather than reasoning, and is mainly concerned with practical purposes.

Keywords : polemic, the art of speech and the art of writing, authority, evidence, reason. 
ZuSAMmENFASSUNG : Kann der Begriff einer polemischen Vernunft bestehen? Dieser Begriff wird hier auf die Probe gestellt, indem man den philosophischen Stil von John Toland zu bestimmen versucht. Dieser Stil, der zu gleicher Zeit eine Denkund eine Schreibart ist, wird in seinen Voraussetzungen sowie in seinen Ausdrucksformen erforscht. Es wird deutlich, daß bei John Toland diese polemische Vernunft, die den Kampf gegen die religiöse Intoleranz aufnimmt und stets auf die Kommunikation zwischen den denkenden Menschen bedacht ist, mehr zu einer Art von Predigt als zu der Beweisführung gehört, und daß ihre Zwecke haupsächlich praktisch sind.

Stichwörter : Polemik, das Mündliche und das Schriftliche, Kommunikationskunst, Autorität, Offensichtlichkeit, Vernunft.

Michel MALHERBE, né en 1941, est ancien élève de l'École normale supérieure, docteur d'État et professeur de philosophie à l'université de Nantes. Il poursuit ses recherches dans le domaine de l'empirisme.

Adresse: Université de Nantes, Département de philosophie, Chemin de la Sensitive du Tertre, B.P. 1025, 44036 Nantes Cedex 01. 
L'idée que la philosophie soit aussi une affaire de style peut choquer le sérieux avec lequel on philosophe aujourd'hui. Les auteurs classiques avaient moins de pudeur et, peut-être, plus d'intelligence. Certes, le style est affaire d'écriture ; et il appelle à ce titre des considérations rhétoriques qui paraîtront étrangères. Mais c'est aussi affaire de philosophie, si l'on admet que non seulement peuvent varier les genres d'écriture, mais aussi bien les modes de penser. Il faut pour cela, et c'est ce qui suscite la plus grande résistance, accepter l'idée que la raison, ou la raison lettrée (comme Bacon parlait d'experientia literata), est plurielle et qu'elle peut adopter des comportements divers.

Cette considération préliminaire ne manque pas d'utilité, quand on aborde les écrits de John Toland. En effet, la seule forme littéraire à n'être pas représentée chez ce polygraphe impénitent est incontestablement la forme académique. Or, si l'on s'interdit de considérer que l'œuvre est irréductiblement disparate ou d'invoquer les seules raisons biographiques (et elles ne sont pas négligeables quand il faut comprendre ce singulier auteur), il n'est pas inutile de tenter de rechercher un dénominateur commun à cette variété littéraire. En ce sens, nous nous proposons d'y discerner un genre philosophique original, celưi de la raison polémique, c'est-àdire d'une raison qui se porte à sa fin obligée - la recherche de la vérité - en soumettant ses procédures aux contraintes d'une relation polémique avec les autres esprits.

Au premier abord, une telle notion paraîtra peu recevable, sinon contradictoire, la justification morale la plus ordinaire de la pratique qu'ont les hommes de la raison étant la concorde et la communion qui doit en résulter. Mais on peut la prendre au moins à titre problématique et, en travaillant les écrits de Toland, essayer d'en dégager les traits et d'en éprouver la viabilité. Sans doute, un tel exercice a-t-il ses limites : une part de reconstruction est inévitable, puisque Toland, qui est pourtant un auteur assez. conscient de son écriture, n'en traite jamais d'une manière didactique; en outre, la diversité de l'œuvre doit être acceptée (même si on la limite aux textes «philosophiques »). Mais prenons le risque de caractériser un concept énigmatique à partir d'une cuvre qui ne l'est pas moins.

\section{I. - LA FORME DE LA POLÉMIQUE}

S'interroger sur les styles rationnels, c'est rappeler que la raison est un commerce, un échange entre les esprits. On l'oublie à trop pratiquer le 
genre de la raison didactique qui s'exprime par traité, par exposé systématique, et qui tire parti de la relation du maître à l'élève : relation d'autorité qui prétend à la neutralité, puisque le maître entend dériver cette autorité de la nécessité même d'une raison véridique dont la validité universelle s'impose tant à lui-même qu'à son élève ou qu'à tout autre. Mais d'autres genres sont possibles qui, plus marqués, rendent plus lisibles la nature de l'échange : la raison dialectique, la raison dialogique, la raison sceptique, la raison facile, etc. et, pourquoi pas, la raison polémique.

À la vérité, cette dernière est en mal d'échange, puisque la controverse qu'elle nourrit en son sein ne peut même pas s'autoriser du consensus minimal que suppose tout dialogue, fût-ce sur le mode du quiproquo, ni prétendre s'efforcer à un accord. Ici, point de paix attendue, où les vérités retrouveraient leur cours ordinaire, leur développement nécessaire et universel. La polémique entretient avec art l'état de différence qui règne naturellement entre les esprits. "This is not a system of accomodation, such as those which some invent to reconcile other different systems, tho they are not certain that their own is more true than the rest ${ }^{l}$. Le consensus, dit Toland, quand il se fait et prétend témoigner d'un fondement assuré, n'est en fait qu'un tissu composite de raisons probables; si jamais il s'impose avec force, s'il prend une tournure officielle, c'est alors qu'on l'appuie sur le préjugé. Car le préjugé est le facteur le plus puissant de la communication entre les hommes : par lui se forment, au fil de l'éducation, ces notions, ces vérités reçues et ces comportements typiques qui rendent l'insertion de chacun plus aisée dans la vie intellectuelle et sociale. Nul n'échappe à son empire, puisque Serena, malgré son rang princier et sa grande culture, avoue ne point réussir à s'en libérer totalement.

Dans la polémique, l'auteur (ou le locuteur) s'avancera donc en position singulière pour briser avec le sens ordinaire des uns et des autres, pour s'affranchir de toutes les ententes préalables. Semblablement, la thèse promue doit avoir un caractère de nouveauté tranchée : elle heurte; on ne fera pas grand-chose pour en atténuer le tranchant ${ }^{2}$. Quant au discours, du moins dans ce moment initial de la prise de parole, il aura la forme du prononcement, de l'assertion catégorique, qui ne cherche pas à s'autoriser

1. John Toland, Letters to Serena (cité par la suite comme Serena), Londres, Bernard Lintot, 1704, lettre IV, $\$ 17$.

2. ID., Nazarenus (cité par la suite comme Nazarenus), trad. franç. Le Nazaréen, ou le christianisme des juifs, des gentils et des mahométans, Londres, 1777, p. XXXIV : «Un des crimes dont ils [les prêtres mercenaires] ont coutume de charger l'auteur qu'ils attaquent est celui d'innovation, laquelle souvent paraît être très recommandable, d'autant plus qu'il est juste qu'une nouvelle réforme prenne la place d'une vieille erreur. Il arrive souvent que ce qu'ils appellent nouveauté est réellement quelque ancienne vérité surannée et hors de mode, mais qui n'est pas moins une nouveauté dangereuse pour des gens dont toute la fortune n'est fondé que sur l'erreur. » 
d'une raison préalable, mais qui tire parti de cette autorité formelle qui appartient au locuteur ou à l'auteur et qui lui est concédée aussi longtemps qu'il détient la parole. Ainsi, sans ménagement et sans ambiguïté, notre auteur se déclare-t-il au début de Christianity not Mysterious ${ }^{3}$, la force et la simplicité de son assertion étant censée disperser toutes les obscurités régnantes et renforcer les doutes qu'entretiennent déjà les esprits sincères :

"On the contrary, we hold that reason is the only foundation of all certitude; and that nothing reveal'd, whether as to its manner or existence, is more exempted from its disquisitions, than the ordinary phenomena of nature. Wherefore, we likewise maintain, according to the title [titre qui donne en même temps le plan de l'ouvrage] of this discourse, that there is nothing in the Gospel contrary to reason, nor above it ; and that no Christian doctrine can be properly call'd a mystery. »

Il serait incontestablement difficile d'être plus franc (et plus imprudent) : il s'agit d'une véritable profession de vérité, chargée de bousculer par un raccourci brutal les interminables débats sur les rapports de la raison et de la religion. Autre exemple : dans la dernière dissertation des Lettres à Serena, l'Auteur (Toland), ne prenant comme précaution que de se réclamer de l'amitié de son correspondant, pose sa thèse que le mouvement est essentiel à la matière, autant que l'étendue et l'impénétrabilité, avec une audace qui défie le sentiment dominant des newtoniens et fait naître dans l'esprit des théologiens quelque anticipation inquiète : I hold then..., I deny that... La vérité présentée ne s'appuie initialement que sur son propre caractère libérateur, lui-même supporté par la singularité presque emphatique de l'Auteur ${ }^{4}$. Et au moment où elle est, elle n'a pour garantie que la sincérité de celui qui la promeut : «I assure him [the well-meaning Christian] that I write with all the sincerity and simplicity imaginable, being as throughly convinc'd of what I maintain, as I can be of anything " ${ }^{5}$.

Il y a certes un danger à mettre ainsi en avant, sur un mode conflictuel, la singularité des locuteurs, le danger de la polémique personnelle. Mais Toland est parfaitement clair sur ce point. Assurément, la singularité qui s'affiche de la sorte en se liant à la thèse proclamée (Toland dit : opi-

3. ID., Christianity not Mysterious (cité par la suite comme Christianity), Londres, 1696, p. 6.

4. Même procédé, presque tonitruant, au début de J. Toland, The Primitive Constitution of the Christian Church, in A Collection of Several Pieces of Mr John Toland, Londres, 1726, vol. II, p. 122.

5. Christianity, préf., p. X. Toute la préface de Christianity est écrite dans cet esprit. Toland insiste également sur le fait que le lecteur ne doit pas lui en faire penser plus qu'il n'en dit, et qu'il n'y a pas de double discours dans ses paroles. Il faut se garder de tenir ces présentations comme de simples artifices visant à atténuer l'effet scandaleux anticipé des thèses défendues. Ni psychologiquement, ni littérairement, Toland n'appartient à l'espèce des gens prudents. 
nion) s'expose à la polémique, à la discussion, sinon à la correction, puisque la thèse en question n'a initialement pour support que le verbe du locuteur qui en assume la responsabilité et qui en est comme le répondant.

"Nor can it appear strange that I should insist upon these terms /Scripture and reason], since I most readily submit my self to them, and give all the world the same right over me [...]. If the reasons I offer be not cogent, I shall take in good part a modest and pertinent animadversion ${ }^{6}$.

Et tous ceux qui entrent ensuite dans la polémique se comporteront de même. Cependant, tous les acteurs du débat méritent le respect : l'adversaire n'est bon adversaire que s'il est traité par son discours et que si lui sont reconnus le courage et la sincérité que suppose la prise en charge de la vérité.

"To reflect on men's persons when we oppose their errors is not only expressly contrary to the sacred precepts of the Christian Religion, but also to the common dictats of reason and below the dignity of human nature $»$ ?

Si elle dégénère en attaque personnelle, la polémique perd son ressort. Bien au contraire, faut-il toujours respecter le devoir de civilité et préjuger de la bonne intention de l'autre, de son honnêteté : «this is the practice of those who really love and esteem the truth, who labor for the reformation and not the destruction of their adversaries ${ }^{8}$. C'est celui qui a l'esprit prévenu par les notions communes et les vérités acquises qui, rencontrant une thèse adverse, s'en prend à la personne, car il lui faudrait autrement interroger sa propre certitude. Alors,

"our first question is not what a man has done or how, but who he is or whence he is?, being ready to approve or condemn, to read over the book or to throw it away, according to the faction or party he espouses"

6. Christianity, préf., p. XVI-XVII. Cf. Nazarenus, trad. franç., p. XLII : « J'ose donc bien exposer ma croyance vis-à-vis de quelque homme que ce soit, si elle est juste il peut s'en laisser persuader, si elle est fausse, il peut m'en convaincre. "

7. J. Toland, Vindicius liberius : or Mr Toland's Defence of Himself... wherein Certain Passages of a Book Intitul'd "Christianity not Mysterious » Are Explained (cité par la suite comme Vindicius liberius), Londres, 1702, p. 6-7. La préface du Nazarenus, trad. franç., p. XVIII-XX, fait le catalogue des mauvais procédés par lesquels on transforme une polémique en procès de l'opposant. Cf. les citations fournies par Stephen H. DANIEL, in John Toland. His Methods, Manners and Mind, Kingston/Montréal, Mc Gill-Queen's University Press, 1984, p. 51 : Vindicius liberius, p. 149; Socinianism Truly Stated, p. 6. Spinoza luimême, si l'on peut contester la solidité de son système, doit être respecté dans sa personne, cf. Serena, lettre IV, \& 3: «I grant you likewise that he was truly sober, observant of the laws of his country and not possest with the sordid passion of heaping up riches [...] I further agree with you that Spinosa's adversarys have gained nothing on his disciples by the contumelious and vilifying epithets they bestow on his person for the sake of his opinion. »

8. Ibid.

9. Serena, p. 15. 
Inversement, qui s'offre à la polémique s'engage à recevoir la critique. Il s'ensuit que la fin de la polémique n'est pas de vaincre l'adversaire, de le réduire au silence ou de le tourner en ridicule, mais bien de soumettre la thèse avancée à son attaque, à son épreuve, pour éviter d'en faire un préjugé, et d'engager un combat de raison, à la loyale, si l'on peut dire. Exercice difficile. Et l'on sait que Toland, à force de s'appliquer à la polémique dans les coffee-houses, avait fini par attirer sur sa personne même les violences verbales de certains de ses opposants; on se souvient aussi comment à partir des années 1705 î en vint, plus prudemment, à écrire à distance, du fond d'une retraite sise à la campagne. En 1704, il rappelle que le sage sait rester indifférent à de telles attaques personnelles, puisqu'il peut juger des passions qui animent l'adversaire. Les hommes de sens «judg of things as they are in themselves, and not as represented to them by passionnate and unjust antagonists ${ }^{10}$.

Ainsi les personnalités engagées dans la polémique ne sont-elles pas des singularités empiriquement déterminées. Le locuteur et l'interlocuteur, l'auteur et le lecteur sont des rôles de langage et de vérité, et représentent en quelque sorte l'enracinement du conflit des thèses dans l'échange verbal, lequel ne peut pas être occulté. Plus précisément, le risque étant que le jeu du langage dégénère en un artifice thêâtral, faut-il considérer que les uns et les autres soutiennent avec sincérité les thèses qu'ils défendent, qu'ils payent en ce sens de leur personne, et que leur personne est ici une intention de vérité assez actualisée pour se produire dans un espace public. Certes, il est difficile de gommer toute détermination empirique : le caractère provoquant d'une thèse nouvelle est ordinairement dépendant d'une certaine situation historique, d'un certain état de la pensée et de la culture. Par ailleurs, lorsqu'il assume dans ses préfaces le rôle de l'auteur, Toland n'hésite pas à personnaliser le Je qui parle par des traits appartenant à sa propre existence ${ }^{11}$. Enfin, il convient de traiter les hommes tels qu'ils sont, et non tels qu'ils devraient être selon un mode idéal de communication. Mais, de quelque façon que la polémique s'enrichisse, ces traits empiriques ne peuvent jouer de rôle moteur dans le débat. Et on doit à Toland de reconnaître que, dans ses écrits les plus importants, il s'attache à s'abstenir de toute critique personnelle, prenant volontiers son parti de cette catégorie de personnes avec lesquelles on ne peut entrer en polémique, parce qu'elles ont condamné avant même d'avoir entendu ou débattu.

10. Serena, lettre IV, \& 3.

11. Cf. la préface de Christianity, p. IX, qui justifie la probité intellectuelle de l'auteur par l'histoire religieuse de sa jeunesse; voir aussi la preface des Letters to Serena qui comportent des allusions claires aux controverses ayant accompagné le Christianity not Mysterious. 
Dès lors, si la fin de la polémique n'est pas la victoire sur l'adversaire, convient-il de la placer dans la recherche de la vérité. La polémique ne peut rester stérile ni se résumer à un mode de communication paroxistique; elle doit avoir un aboutissement : l'établissement d'une vérité libre de préjugé et de superstition. Cet établissement doit être conflictuel, afin que soit évitée la fondation d'une secte nouvelle au nom d'un système nouveau. C'est là que fut le défaut de Spinoza qui était «too much in love with his new world $»^{12}$. Le sectarisme tire, ou prétend tirer, sa force de vérité de la nouveauté de sa thèse; mais il incline au dogmatisme, car il transforme sa thèse en un système ayant réponse à tout, quelque impuissant qu'il soit à couvrir tout le champ de la vérité, et n'ayant de recours pour compenser cette faiblesse que d'afficher une forme systématique. Au contraire, on ne saurait porter d'accusation contre quiconque

«makes some particular discoverys or who even changes the whole face of philosophy, and introduces a method absolutely new; for such persons may without all question be acted by no other motives besides the love of truth and the benefit of the society nor will they reject anything but what they really conceive to be hurtful, erroneous or unprofitable ${ }^{13}$.

Peut-être trouvera-t-on impossible que le conflit frontal (et non dialectique) des thèses mène à autre chose qu'à la victoire ou à une guerre sans fin entre les personnes ou entre les systèmes. Peut-être trouvera-t-on paradoxal, alors que l'on a fait de la concorde, du consensus, un attribut du préjugé, de prétendre encore atteindre à la vérité : la polémique ne vit-elle pas du caractère irréconciliable des opinions et des thèses?

Assurément, si le débat tend vers une issue dogmatique ou, même, plus modestement, vers un consensus composé, l'entreprise est vaine : jamais d'une polémique on ne tirera une unité d'opinion. En fait, chez Toland, le ressort de la vérité est ailleurs.

Prenons, en guise d'exemple, la thèse de la seconde lettre à Serena, selon laquelle la notion de l'immortalité de l'âme n'est pas venue aux païens par le biais des juifs (c'est-à-dire par quelque révélation divine) ni par le moyen de raisonnements philosophiques, mais est dérivée des rites et des pratiques mortuaires des Égyptiens. Un tel propos ne peut manquer de provoquer théologiens et philosophes : il y va de la puissance de la Révélation (seule source, dit Toland, de cette croyance pour les chrétiens) ou de la Raison (source bien faible, dit Toland après Cicéron, pour entretenir une croyance aussi forte). Mais, ajoute notre auteur, la question du fondement d'une vérité si utile a été et sera un objet de débat et de contestation sans

12. Serena, lettre IV, $\S 6$.

13. Serena, lettre IV, $\S 5$. 
fin. Or, si la présente thèse participe elle-même de ce débat, elle recourt néanmoins à un procédé différent, celui que lui offre la méthode historique et exégétique. L'origine égyptienne est un fait attesté par les textes antiques que l'on peut soumettre à une analyse critique. On débattra donc toujours du véritable principe de l'immortalité de l'âme; peut-être discutera-t-on par ailleurs le détail des faits; mais on peut s'accorder sur la nécessité d'employer la méthode critique. La thèse défendue peut donc prévaloir, si l'on rapporte la recherche de la vérité non à un point de doctrine, mais à un point de méthode.

Soit encore la réfutation de Spinoza. Toland déclare ne pas vouloir entrer dans la critique des thèses particulières de son prédécesseur, mais faire porter l'examen sur le tout du système. Or la faute majeure de Spinoza est une faute de méthode, qu'il convient de reconnaître. Si, au moment d'établir ses principes, le philosophe hollandais n'a pas su attribuer à la substance première le mouvement au même titre que l'étendue et la pensée, si de la sorte il a rendu impossible la détermination de la matière en figures déterminées, se contentant du mouvement local (considéré comme définissable par l'étendue), c'est parce que, tout en prétendant suivre la méthode géométrique, il a confondu le mouvement local et la force active (par là même ignorée), c'est-à-dire il a confondu l'effet et la cause, la conséquence et le principe. "Most of the difficultys you mention, proceed from peoples confounding the cause with the effect, or the moving force with local motion ${ }^{14}$. La polémique, ici encore, permet de dégager un instrument critique qui est soumis au lecteur.

Assurément, un éventuel accord sur la méthode ne suffit pas à réconcìlier les opinions ni à tarir la polémique. D'autant que les vérités auxquelles l'on a affaire sont souvent des vérités pratiques (morales, politiques ou religieuses). Car, d'une façon générale, toute vérité importe aux hommes; attribuer le mouvement à la matière n'est pas qu'une thèse spéculative, et la résistance qu'on lui oppose tient largement aux conséquences pratiques que l'on peut en tirer. Opposition qui ne saurait être réduite à rien, puisqu'il y va de la vie humaine ${ }^{15}$. Plus que du conflit des vérités, la polémique se nourrit du combat militant des valeurs auxquelles les hommes tiennent parce qu'elles leur importent, soit qu'ils les valorisent d'eux-mêmes, soit qu'elles leur aient été inculquées. C'est la raison pour laquelle la vérité est toujours vérité exposée, défendue ou précipitée. Mais s'il est impossible, et même non souhaitable, de suspendre les opinions des hommes ou de les

14. Serena, lettre IV, $\$ 14$.

15. Serena, lettre III, $\$ 22$ : la recherche de la vérité ne saurait être animé par la seule curiosité : "nor in my opinion, is the gratifying of mens curiosity a sufficient recommendation to any disquisition, without some general instruction naturally conducing to wisdom or virtue. \ 
noyer dans l'impassibilité de la simple spéculation, on peut néanmoins les rapporter à une méthode d'analyse ou de critique sur laquelle on peut s'entendre.

Au demeurant, le philosophe lui-même est un homme. Si la vérité (ou ce qui passe pour tel) a de la valeur pour lui, pour sa vie, inévitablement, il se hâte de la penser, de la faire valoir, de la revendiquer, et même de l'appliquer. Il préjuge et il se précipite. D'où l'extraordinaire puissance et constance du préjugé ou de la superstition.

«Thus the body of the people in all places of the world do greedily imbibe whatever they are taught to imitate or to respect from their infancy, and without further evidence are ready to die for the truth of it in old age $\gg^{16}$.

"In all times superstition is the same, however the name of it may vary, or that it may have different objects, or be greater or less in degrees, as any country has more or less liberty of conscience and free speech $" 17$.

Se soumettre soi-même à la controverse est donc le moyen de s'affranchir ou de résister au conformisme du préjugé, à l'influence d'autrui, à l'état d'ignorance qui entoure les passions, et de tenter d'accéder à une position critique. L'affichage polémique d'une thèse est le moyen pour celui qui la prononce d'imposer à ses certitudes du délai, de soumettre son adhésion, qu'il ne suspend pas, à l'épreuve de la critique, et de la sorte de ne pas tomber dans l'esprit de système, dans la défense sectaire.

\section{II. - LE PROBLÈME DE L'ÉCRIT}

La polémique est par essence publique et son bruit dépasse les portes des coffee-houses. La rumeur s'en saisit et la déforme; de là naissent les actes d'intolérance envers les personnes. La polémique n'a pas alors atteint ses fins. Mais il en va ainsi de tout espace public ouvert. Socrate n'enseignait pas aux foules, mais fut bien condamné à mort. Et dans l'Antiquité, face aux préjugés religieux, par crainte que la vérité ne fût persécutée, désespérant d'une réforme collective, les philosophes en vinrent à limiter la communication à un petit nombre, sous le couvert d'une obscurité protectrice.

«And therefore, when Anaxagoras discover'd that the moon had but a borrow'd light from the sun, and so gave the reasons of its wax and wane, such a

16. Serena, lettre I, $\S 11$.

17. Serena, lettre III, \& 22 . 
doctrine durst not be made publick, but was secretly communicated to very few, and even to them under a promise of fidelity ${ }^{18}$.

De même, en matière religieuse :

«All those therefore who had penetration enough to discover and courage to oppose the folly or craft of this [polytheist] theology, were call'd and reputed atheists [although pure theists] and treated as such by the multitude at the instigation of the priests $\gg$.

Bref, alors que, pour échapper à l'esclavage du préjugé auquel la multitude reste soumise, ils auraient dû pratiquer la raison polémique, qui est éminemment publique, les anciens philosophes renoncèrent à être courageux et s'adonnèrent à une communication ésotérique, afin de préserver le confort de la philosophie.

Quant aux philosophes modernes, ils prennent le parti de se retirer à la campagne et de limiter leur commerce à des voisins qui soient gens de sens et de bien ${ }^{20}$ ou à des correspondants qui sont restés en ville. L'écrit remplace alors le verbe. L'écrit permet de se dégager de la circonstance ou de la menace, il allège le poids du public et garde de l'intention pamphlétaire. C'est pourquoi, dans sa biographie de James Harrington ${ }^{21}$, Toland demande qu'on ne retienne de cet auteur que les écrits durables ayant une valeur pour la communauté humaine, et non ces écrits de circonstance ou de polémique vive qui ne méritent pas de durer plus que ce qui les a motivés. Mais l'écrit peut-il faire place à la raison polémique?

La forme littéraire qui sera privilégiée est celle de la lettre, dont on sait que Toland a fait un usage considérable, puisque même l'Histoire des druides prend le tour d'une correspondance adressée à Molesworth. Il est vrai, la forme littéraire peut n'être qu'un artifice d'exposition, une recherche de patronage, un procédé d'atténuation ou d'insinuation, de manière à servir l'esprit dogmatique aussi bien que l'esprit polémique. Mais, on observera avec quelle aisance Toland pratique la forme épistolaire, avec quel naturel il nous fait douter s'il s'agit d'une fiction ou d'un échange véritable se poursuivant par écrit, de sorte qu'il en résulte une incontestable impression d'authenticité.

La lettre permet l'ouverture d'un espace de communication à la fois plus large et moins engagé et une liberté de propos plus considérable. Mais elle conserve les traits généraux de la raison polémique. D'abord la conscience

18. Serena, lettre III, $\S 18$.

19. Serena, lettre III, $\S 18$.

20. Serena, lettre IV, \& 1 .

21. J. Toland, "The Life of James Harrington ", in James Harrington, Works, Londres, 1771, repr. Aalen/Darmstadt, Scientia Verlag, 1980, p. XXIV. 
d'originalité : l'auteur est censé souvent répondre à une demande de son correspondant, soit réagissant à un propos singulier tenu antérieurement par l'auteur, soit s'interrogeant sur un sujet d'importance qui invite à fuir la banalité des idées reçues. Ensuite, l'ouverture à un échange virtuel est maintenue; non pas que le correspondant soit un adversaire direct (la lettre renforcerait alors le tour personnel de la polémique) : il est soigneusement distingué de ceux auxquels on ne peut même pas s'adresser, puisqu'ils ont prévenu par leur intolérance toute relation possible; mais il n'est pas réputé épouser les sentiments de l'auteur, il peut même faire objection. En fait, le correspondant est un intermédiaire entre la campagne et la ville, entre l'auteur dans sa retraite, qui a pris de la distance avec l'esprit de querelle, et le public, dont on ne sait s'il est favorable ou non.

Dans les Lettres à Serena, l'œuvre certainement la plus travaillée à cet égard, on remarque la diversité des correspondants : si les trois premières lettres sont adressées à Serena, dans laquelle chacun reconnaît SophieCharlotte, la reine de Prusse qui trouvait plaisir à converser avec Toland, les deux dernières sont adressées l'une à un gentleman hollandais partisan de Spinoza, personne de grande probité intellectuelle ${ }^{22}$, l'autre à un autre gentleman ami du précédent, et levant des objections contre l'idée que la matière est active; deux correspondants auxquels on serait tenté par symétrie d'accorder une existence réelle anonyme, Serena étant le nom d'un personnage historique réel.

S'il est vrai que Sophie-Charlotte n'est pas en position adverse, son rang princier et son intelligence réputée interdisent que l'auteur la traite sur un mode autoritaire. Toland répond à sa demande et lui soumet ses analyses historiques et critiques. Par ailleurs, écrire à une reine, c'est vouloir assurément rendre la correspondance publique. Mais la préface qui présente les cinq lettres est à son tour adressée à un gentleman. Ce gentleman a des angry friends $^{23}$; et l'auteur laisse la liberté à son correspondant de montrer ces lettres à qui il veut, ami ou ennemi ${ }^{24}$; il ne préviendra pas l'emploi négatif qui pourrait être fait de son discours, dont le contenu, dit-il, ne comporte rien qui soit propre à entretenir les passions religieuses et politiques. «They are only innocent researches into the venerable ruins of Antiquity, or short essays in philosophy, not calculated to offend any, but to please all; and to divert, if they are not capable of instruct $"{ }^{25}$. Cette multiplication croisée des destinataires (alors que l'unité thématique du tout est aisément perceptible par le lecteur) permet de lier des développements variés à la fois par leur

22. Serena, lettre IV, § 14.

23. Serena, préf., $\$ 16$.

24. Serena, préf., $\$ 15$.

25. Serena, préf., $\$ 15$. 
nature et par leurs conséquences et, incontestablement, d'assurer une réelle liberté de propos ${ }^{26}$. Le ton sera serein et dépourvu de passion.

S'il est clair que la forme épistolaire favorise l'échange des opinions, il peut paraître moins évident qu'elle convienne à la raison polémique. Mais il faut introduire un dernier acteur : le lecteur lui-même. Pas plus que le rapport au destinataire, le rapport au lecteur n'est neutralisé; car ce dernier n'est autre qu'un destinataire plus lointain, moins lecteur anonyme ayant acheté le livre chez un libraire, que lecteur appartenant à l'entourage de l'un des correspondants. On lui attribuera par principe tolérance et bienveillance. Mais on ne lui déniera pas le droit d'entretenir ses propres opinions, lesquelles peuvent concorder ou non avec celles de l'auteur. Et l'adresse qui lui est faite ne laissera pas d'être d'essence polémique dans la mesure où, sous l'échange courtois et philosophique qui est de règle, l'auteur s'efforce de promouvoir des thèses dont il connaît le caractère singulier, sinon provoquant ${ }^{27}$. Par exemple, l'auteur n'ignore pas qu'en faisant la généalogie de la croyance païenne en l'immortalité de l'âme, il est susceptible de toucher les sentiments religieux ou philosophiques du public, lequel ne manquera pas de réagir, puisqu'il ne faut pas être grand clerc pour comprendre que, d'un côté, au moment même où l'on en appelle à la Révélation pour instruire les hommes de cette importante vérité, on place en concurrence une histoire naturelle de la croyance en l'immortalité de l'âme, c'est-à-dire l'histoire d'un préjugé, d'une superstition; et que, de l'autre côté, on interdit toute justification rationnelle, puisque précisément l'on en appelle à la Révélation. Mais c'est au lecteur qu'est laissé le travail de rapporter la thèse défendue à ses propres opinions ${ }^{28}$. Tant par la liberté formelle offerte par la tournure épistolaire que par la liberté réelle accordée au lecteur d'avoir une opinion ou de se faire une opinion, la raison polémique se garde de déterminer autoritairement la nature de la réception de la thèse.

\section{III. - AUTORITÉ, ÉVIDENCE ET RAISON}

Les philosophes modernes n'aiment pas l'autorité. D'aucuns veulent que la philosophie laisse parler les choses mêmes ou qu'elle épouse le déve-

26. Dans Adeisidaemon, sive Titus Livius a superstitione vindicatus, La Haye, 1709, J. TOLAND recourt au même procédé : la préface est une lettre adressée à Anthony Collins et présente le texte des deux lettres qui font le contenu de l'ouvrage : la première sur Tite Live est adressée à un prêtre de l'Église anglicane; la seconde à un certain Augustus Samsonius, lequel a fait part de ses réactions sur le contenu de la première lettre.

27. Cf. Serena, préf., $\S 12:$ l'annonce de la troisième lettre qui exposera " the origin of idolatry, explained after a very different manner from what is commonly received».

28. Serena, préf., $\$ 15$. 
loppement de l'Esprit, qu'elle laisse parler la parole qui l'occupe, etc. D'autres, au contraire, veulent qu'elle se soumette au formalisme des règles de la logique. Ici et là, point d'autorité apparente; rien d'officiel sinon le droit de la vérité ou de la règle. Or toute communication quelle qu'elle soit (et l'échange philosophique ne fait pas exception) requiert plusieurs types d'autorité : autorité du locuteur qui prononce verbalement; autorité du maître, de l'écrivain, de l'orateur, etc., qui prend appui sur une forme institutionnelle ou sociale pour assurer la transmission; autorité de la thèse qui tire parti de sa force de déclaration et d'élucidation; autorité d'une méthode qui est privilégiée au détriment de quelque autre; autorité absolue du fait que l'on produit; autorité des représentations et des raisons qui imposent leur validité.

La raison polémique a ce mérite d'incorporer dans son procédé l'indispensable traitement de l'échange; à ce titre, elle ne recule pas devant l'autorité, mais, en quelque sorte, elle la démultiplie. Tandis que la raison dogmatique confond en une seule détermination la rigueur des raisons, le poids des faits, la prise de parole, le pouvoir institutionnel et par là gagne en intolérance, la raison polémique, quant à elle, s'autorise du verbe de l'auteur, mais pour le référer à un destinataire; elle n'hésite pas à prononcer, à déclarer la thèse, lui conférant autant de force publique que possible, mais pour susciter la controverse; elle en appelle aux faits, mais l'établissement des faits dépend de l'emploi d'une méthode; elle en appelle à la méthode, mais aucune méthode ne l'emporte absolument; elle en appelle aux raisons, mais les raisons sont valides ou non pour des esprits qui les reçoivent ou les refusent ou les discutent.

Pour Toland, la fin recherchée est donc la vérité (et non l'attaque personnelle ou la suspension sceptique), et le moyen retenu est un ensemble de procédés tant rhétoriques qu'intellectuels visant à briser l'intolérance, qu'elle se réclame d'un dogme, d'une secte ou d'une révélation. En quoi ces procédés permettent-ils d'atteindre à la vérité et quelle est leur essence rationnelle, s'ils en ont une?

Selon la grande thèse métaphysique de Toland, le mouvement est un attribut de la matière, laquelle est censée renfermer une force active éternelle. Dans la quatrième des Lettres à Serena, cette thèse est introduite comme la conséquence de la réfutation de Spinoza. En vérité, la conséquence est certainement le motif de la réfutation, puisque Spinoza, quoique attaqué, joue le rôle fondamental d'introduire une substance universelle dotée d'attributs dont les êtres singuliers sont autant de modes. Mais le philosophe hollandais, lui reproche-t-on, n'a conféré à la substance universelle que les attributs de la pensée et de l'étendue; et, en ce qui concerne les corps, il se montre incapable de rendre compte des figures déterminées de la matière et, par extension, des êtres séparés qui 
composent le tout de la nature. Or, il faut rendre compte du mouvement éternel, sans cesse déterminé, par lequel surgissent, se maintiennent ou se perdent les corps dans leur immense variété.

Toland met alors en place le dispositif polémique. Le correspondant est censé demander à l'auteur son « opinion particulière »; et l'auteur ne se dérobe pas : il affiche sa thèse, sans prendre beaucoup de précaution ni la préparer par les raisons qui seraient susceptibles de la justifier (lesquelles ne viendront pas dans cette quatrième lettre). Les risques encourus sont assumés : " a man is very like to be wrong understood who delivers his opinion (especially if altogether new) before it be guarded with its proofs and explication " ${ }^{29}$. Quant à la thèse, elle ne tient encore que par l'effet de sa nouveauté et l'autorité de l'auteur qui la profère. Puis Toland décrit la situation polémique ainsi ouverte ${ }^{30}$ : l'auteur doit-il redouter de ruiner tant de doctrines et d'hypothèses adverses et de se créer ainsi un grand nombre d'adversaires? «To this I answer, that the offence is taken, but not given; and that I shall thereby be nothing disquieted, provided I be able to contribute any thing towards the discovery of truth. "

Le premier gentleman se contentera peut-être d'avoir perdu ses sentiments spinozistes pour épouser la nouvelle thèse. Mais le second réclame des preuves dans la dernière lettre. Et l'auteur y consent.

«As our opinion ought to go for nothing without good reasons, so we admit of no right from possession, of no privilege by prescription in philosophy. how much soever we allow it in national laws or customs. Authority is to decide matters of fact, but not to determine the truths of nature ${ }^{31}$.

Or l'auteur annonce le procédé remarquable, mais conforme à une stratégie polémique, qui va être le sien : comme son correspondant considère la thèse comme paradoxale et absurde, l'auteur s'appliquera à reprendre littéralement chacune des objections que son adversaire a formulées, pour les réfuter. "For my intention has not bin to write all I could say on this subject, but as much as I thought necessary to answer your objections, and to bring you over to the same opinion $"{ }^{32}$. Aucune démonstration directe de la thèse ne sera donc apportée; mais toute la preuve attendue consistera à récuser les objections qui lui sont opposées ${ }^{33}$. En ce sens, le débat porte

29. Serena, lettre IV, \& 16.

30. Serena, lettre IV, \& 17 .

31. Serena, lettre V, \& 1 .

32. Serena, lettre V, $\S 31$.

33. La même technique est employée de façon dominante dans Christianity, en part. au début, section I, p. 1-6, quand il faut établir la rationalité de l'Évangile. Tous les arguments invoqués pour justifier l'existence de mystères dans la religion chrétienne sont passés en revue, avant que ne soit déclarée (et par là justifiée ou, du moins, motivée) la thèse de l'auteur. Même procédé dans la section II, quand il faut établir que la religion n'est pas contraire à la raison : les différentes variantes de la thèse adverse sont poussées à leurs conséquences intel- 
directement non sur la thèse, mais sur les objections; et on laissera à l'adversaire le soin de peser les réponses aux objections, de tenir en son esprit la balance de la vérité et, alors, de se rallier éventuellement à la thèse présentée. Nulle part, la thèse n'est établie par elle-même (elle mériterait évidemment de l'être : on attend toujours, par exemple, que Toland dise ce qu'il entend par mode et par attribut ${ }^{34}$ ).

Les procédés mis en œuvre dans ce travail assez long de réfutation sont remarquables. D'abord, quelle que soit la façon dont l'objection traitée est levée, la thèse, à défaut d'être prouvée, est constamment répétée de façon à ce qu'elle s'implante dans l'esprit du correspondant (ou du lecteur). Ensuite, on peut distinguer deux grandes méthodes de réfutation. D'une part, on fait valoir une sorte de raisonnement par l'absurde, ou plutôt par défaut ${ }^{35}$ : on montre que les autres opinions ne suffisent pas à expliquer les mouvements particuliers qui génèrent les différents êtres qui sont dans le monde; mais sans que les insuffisances en question soient vraiment mesurées ni que le caractère contradictoire entre ces opinions et la thèse soit assez souligné pour conforter la validité de cette dernière. D'autre part, et c'est plus intéressant, la thèse adverse est moins saisie dans son contenu ou ses raisons que dans son statut de proposition erronée dont il faut génétiquement rechercher la cause. Ainsi, du préjugé universellement répandu que la matière est inactive : on a d'abord considéré, dit Toland, que le mouvement était extérieur à la nature, puis on a transformé cette erreur initiale en un préjugé indiscutable, fortement enraciné dans de fausses doctrines. Ainsi encore du concept d'espace ${ }^{36}:$ "I shall give you the history of this opinion as I have done of the rest, tho I might justly neglect it after having proved matter to be essentially active. " Les mathématiciens se sont donné pour objets des notions abstraites, ce qui est leur droit le plus manifeste; mais ce faisant, ils ne se comportent pas en physiciens : l'idée d'espace absolu présentée par Newton et ses partisans est la réalisation indue de ce qui n'a que statut d'abstraction. D'une façon générale, Toland ${ }^{37}$ s'essaie à une classification génétique de toutes les opinions adverses, en faisant varier la thèse du repos absolu qui est la conséquence obligée de l'inactivité de la matière; et il présente ${ }^{38}$ une sorte de catalogue

lectuellement absurdes ou pratiquement inacceptables. La section III, après avoir défini deux sens du mot mystère, écarte successivement tous les sens indus, ceux qui nourrissent l'idée que certaines vérités, quoique non opposées à la raison, lui seraient supérieures.

34. Voir encore la définition donnée des corps comme étant des modifications de la nature ou des quantités particulières, séparées abstraitement par l'esprit, mais non séparées réellement de l'élément de l'univers : cette véritable définition réelle (tout à fait fondamentale pour le propos) est traitée comme une simple définition verbale.

35. Serena, lettre V, par exemple, \&8.

36. Serena, lettre V, $\$ 12,24, s q$.

37. Serena, lettre V, $\$ 23$.

38. Serena, lettre V, § 24 et 25. 
de toutes les causes d'erreurs: les apparences sensibles qui trompent le jugement; la force et la constance des préjugés; la genèse des notions fausses à partir d'une opinion initiale erronée; la mauvaise abstraction. Bref, la réfutation consiste à faire la critique non de la thèse adverse ellemême, mais de l'acte intellectuel qui y a conduit.

L'arme de la polémique apparaît ainsi clairement : elle consiste essentiellement à ne point s'engager dans un argument théorique, raison contre raison, mais à réduire la doctrine adverse par une analyse critique qui permet de la traiter sur le mode du fait et, partant, d'en rendre compte par une analyse génétique. En quelque sorte, on la prive de cette pertinence rationnelle qu'il faut accorder à toute doctrine contraire quand, en vue de la vérité, on installe la controverse dans l'élément de cette contrariété. À cet égard, la polémique est (comme toute polémique) unilatérale : l'adversaire n'est pas entendu, quoiqu'on lui accorde formellement le droit d'en user de même.

Le procédé était déjà à l'œuvre dans le premier ouvrage de Toland, Christianity not Mysterious. On le rencontre, par exemple, dans la troisième section qui, en écho de l'Essai de Locke, prétend établir qu'il n'y a rien de mystérieux dans la religion chrétienne qui soit au-dessus de la raison (la deuxième section ayant établi qu'il n'y a rien de contraire à la raison). Tout argument réducteur suppose un dispositif initial, assurément non innocent, qui permet d'arracher la question au plan spéculatif sur lequel elle se tient, pour la ramener à une question de fait, étant entendu qu'un fait, à la différence d'une raison, ne se discute pas, mais est ou n'est point (c'est là la force du fait que d'être spéculativement neutre et sans appel). Toland commence par transformer la question du rapport entre la foi et la raison en une analyse des significations du mot mystère. Et il distingue deux sens : tantôt mystère dénote une chose intelligible par elle-même, mais cachée sous le voile des mots et rendue obscure par les pratiques religieuses (rites et cérémonies); tantôt est signifiée une chose qui est censée être par soi inconcevable, mais qui aurait été néanmoins clairement révélée (en sorte qu'il faut y apporter sa foi, quoiqu'on ne la comprenne pas). Toute la stratégie - simple - du texte consiste à établir que seul le premier sens, celui qu'entendaient les païens avant la Révélation et qu'on trouve dans leurs écrits, est assignable, et que le second, celui qui est entretenu aujourd'hui par les partisans du mystère chrétien, n'est pas recevable et qu'il n'est pas reçu dans le Nouveau Testament, lequel ne connaîtrait que le premier sens. En conséquence, Toland ${ }^{39}$ montre qu'on ne saurait masquer l'absurdité du deuxième sens du mot (à savoir que puisse être clairement révélé ce que l'on ne peut penser) en abusant d'un argument philo-

39. Christianity, chap. II. 
sophique selon lequel serait mystère ce dont on ne peut avoir de connaissance adéquate. Il suffit pour cela de rappeler la critique lockienne de la connaissance d'essence et de montrer qu'à ce compte ce serait toute connaissance humaine qui serait mystérieuse. Puis Toland ${ }^{40}$ relève les significations du terme mystère dans le Nouveau Testament, sous deux sens, l'un plus général et l'autre plus particulier : est dit mystère tantôt ce qui, avant la Révélation, avait été caché à toute l'humanité, parce que cela était compris dans le décret divin, tantôt ce qui n'avait été que partiellement caché au peuple juif - la parole du Christ ayant dans les deux cas dissipé l'ignorance et l'obscurité. Et les textes sacrés nomment ainsi mystère l'Évangile lui-même qui ne pouvait être connu des hommes privés de la Révélation, ni même des juifs, ou encore toute doctrine particulière effectivement révélée par les apôtres ou encore les fables, les prophéties, les jeux de discours dont la leçon a été parfaitement clarifiée par le Christ. Bref, il n'y a pas d'essence du mystère, mais seulement une réalité historique qui fut celle des gentils et des juifs, et qui a aujourd'hui disparu, puisque Dieu nous a instruits par sa Parole.

Toute l'argumentation a été concentrée sur l'usage du mot mystère : il suffirait de vérifier son emploi dans la pratique générale des hommes et dans le texte testamentaire. Et la décision sera assurément une décision de fait : on établit, citations à l'appui, que le Nouveau Testament n'emploie le mot qu'au premier sens, qui est le sens régulier. Et comme la Révélation a apporté aux hommes ce qui leur manquait, il s'ensuit qu'il n'y a rien de mystérieux dans le christianisme et que tout y est rationnellement compréhensible. Il n'est pas alors surprenant que, pour terminer et après avoir répondu à une série d'objections, Toland fasse une histoire (reconstituée, plutôt qu'établie sur des faits précis) du second sens du terme et de l'obstination avec laquelle certains prétendent maintenir le mystère comme une part de l'essence de la religion chrétienne et en faire une condition de la foi : les juifs christianisés ont voulu conserver certaines de leurs traditions; les gentils ont introduit leurs rites païens au moment de leur conversion; les philosophes n'ont pas été en reste et ont imposé leurs systèmes et leurs sectes; quant aux politiques, ils comprirent rapidement l'utilité de plonger les esprits des hommes dans le respect de ce qu'ils sont censés ne pas comprendre.

La question des rapports de la foi et de la raison n'a donc pas été débattue : ni philosophiquement ni théologiquement ${ }^{41}$. Il est clair que la polé-

40. Christianity, chap. III.

41. Et pourtant, les postulats théoriques de cette singulière argumentation ne sont pas négligeables. Pour n'en citer qu'un seul : pourquoi la Parole divine ne nous a-t-elle instruits que des faits compris dans le décret divin? Et pourquoi ne nous aurait-elle pas informé des faits du mystère divin? Qu'un fait soit ou totalement ignoré ou totalement connu ne préjuge pas de la nature de ce qui s'y présente. 
mique, ainsi entendue, ne se développe pas en un conflit contradictoire ou dialectique des raisons certaines ou des raisons probables. Remarquablement, il n'y a pas d'affrontement entre thèses opposées, mais plutôt une procédure de disqualification de la thèse adverse.

Mais quand bien même l'on accorderait la méthode employée pour la critique, il reste à considérer la manière dont l'auteur justifie sa propre thèse, puisque, faute d'un rapport de contradiction ou de contrariété développé entre les thèses en présence, l'invalidation de la thèse adverse n'est pas encore la vérité de la thèse propre. Or, sur ce point, un trait remarquable doit être souligné chez Toland. La thèse repose tout entière sur sa proclamation. Ou, pour le dire autrement, elle est montrée, jamais démontrée. Elle est donnée à voir, car elle est censée porter en elle-même son évidence. Évidence originaire, et par conséquent native; évidence également perdue, car l'erreur philosophique, le préjugé de l'éducation ou la superstition religieuse ont éloigné les hommes d'une certitude initiale qui s'imposait d'elle-même et dont on peut retracer généalogiquement la corruption. Toland fait souvent jouer cette autorité d'une évidence retrouvée, autorité qui étant primitive dispense de la preuve, mais qui a besoin d'être confortée auprès d'esprits prévenus. Le Nazarenus, pour prendre cet exemple, est composé de deux lettres qui ont chacune pour motif une découverte bibliographique de Toland : d'un côté, l'Évangile de Barnabé, à replacer dans l'histoire des Nazaréens, de l'autre côté, un manuscrit des Quatre Évangiles écrits en caractères irlandais et témoignant d'un christianisme antérieur à l'influence papale. Or, dans les deux cas, et c'est ce qui fait l'unité de l'ouvrage, est valorisée l'idée d'un christianisme plus originaire et plus pur, dont la religion contemporaine s'est éloignée et dans lequel se reconnaît l'auteur qui se déclare nazaréen ${ }^{42}$. Dès son premier ouvrage, Toland avait valorisé cette fonction de l'évidence :

«It is impossible for us to err as long as we take evidence for our guide; and we never mistake, but when we wander from it by abusing our liberty, in denying that of any thing which belongs to it, or attributing to it what we do not see in its idea. This is the primary and universal origin of all our errors $"{ }^{43}$.

La liberté d'errer est une corruption de la liberté que nous a accordée le Créateur envers ce qui est indifférent, douteux ou obscur. Et si l'on demande comment il peut se faire que l'esprit ne se porte pas aux évidences qui s'imposent à lui, on répondra par les défauts ou les corruptions

42. Sur le même thème, voir J. Toland, op. cit. supra n. 4, vol. II, p. 132 : il évoque un christianisme primitif qui, à la fois dans sa doctrine et dans sa pratique religieuse, n'avait pas encore subi l'influence du culte des juifs, des mystères païens et des arguties des philosophes.

43. Christianity, chap. 1, p. 4, 14. 
qui affectent la transmission de l'évidence entre les esprits (problèmes de mots, problèmes d'ordre d'exposition, quiproquos, prévention, malignité, jeux de pouvoir).

C'est pourquoi, à témoigner d'une évidence perdue et retrouvée, la pratique de la raison polémique relève-t-elle chez Toland du registre de la profession (de vérité, sinon de foi), et même de la prédication. On se dérobe à tout affrontement théorique entre doctrines, on soumet le discours de l'adversaire à un procédé réducteur qui l'annihile en quelque sorte avant même qu'il ait eu le temps de se poser, de se développer rationnellement, et, enfin, en face, pour ainsi dire, on fait rayonner la vérité à promouvoir. Or, une telle démarche, qui est l'antithèse de l'ironie socratique, est, à la considérer d'une façon générale, caractéristique de la défense des valeurs. Et qu'il y ait un militantisme de la part de Toland est manifeste. Devant ceux qui s'en prennent à la philosophie, la confondant avec les systèmes des philosophes, et qui exigent une soumission aveugle de la raison, il s'exclame : "Ridiculous! as if Reason and Truth were vanity and craft " ${ }^{44}$. Religieux et philosophes se sont unis en scolastique pour défendre les mystères incompréhensibles d'un côté et les qualités occultes de l'autre. Ces extravagances « were both calculated at first for the same ends, viz. to stop the mouths of such as demand a reason where none can be given, and to keep as many in ignorance as interest shall think convenient $»{ }^{45}$.

Mais peut-on militer en faveur de la raison elle-même et prétendre la représenter comme une valeur? N'est-ce point précisément aligner son propre comportement sur celui de la partie adverse, pour engager un combat où l'on espère le triomphe?

Les ouvrages postérieurs ont perdu la virulence du Christianity not Mysterious, sans vraiment en détendre le ressort. Tout conflit de valeur peut dégénérer en intolérance, et l'intolérance est incompatible avec la raison elle-même. Comment alors adopter le parti de la raison tout en restant en conformité avec elle? Le dispositif de la polémique tel que nous avons essayé de le décrire est certainement un effort pour éviter une telle conséquence. Au demeurant, l'adversaire que l'on se donne, s'il a des opinions éventuellement arrêtées, est de bonne foi et n'est pas à ce point partisan qu'il s'adonnerait à la violence. Le rationaliste lui-même s'astreint à la sérénité et à la mesure; mieux : il fait effort de preuve. Et Toland ne manque pas d'intérêt dans ses analyses historiques et critiques; son œuvre confirme même un genre philosophique qui est en train d'émerger, celui de l'histoire naturelle. Mais on ne peut manquer de relever la faiblesse majeure d'une telle raison polémique et de toutes les entreprises du même

44. Christianity, section III, chap. $4, \S 46$.

45. Christianity, section III, chap. $4, \S 46$. 
type qui suivront: les deux opérations complémentaires de la critique réductrice et de l'explication génétique, quand elles ne sont pas traitées comme des procédures sceptiques, s'avèrent incapables de fonder la thèse mise en avant qui est ainsi livrée à sa propre puissance d'appel. Que toute philosophie ne soit pas dépourvue de ce tour, cela est certain; et, somme toute, la philosophie n'est qu'un discours humain. Mais on peut attendre d'elle que, précisément, si elle tire de la raison des vérités qui sont autant de valeurs, elle en rende raison autrement qu'en en appelant à la valeur de la raison.

\section{IV. - PAR-DELÀ LA RAISON POLÉMIQUE}

Le Pantheisticon commence par une adresse émouvante. L'épître préliminaire se tourne vers "the learned and ingenious reader» qui est un "candid reader", dans une annonce solennelle: "As one who has the interest of mankind greatly at heart and as a strict votary of the eternal truth, I present to you a new fellowship and new regulation ${ }^{46}$. Pour l'essentiel, le même dispositif rhétorique est encore à l'œuvre. Mais le public est désormais doublement restreint : l'auteur s'adresse à gens instruits, car « the generality of mankind is averse from knowledge, and vents invectives against its partizans $"{ }^{47}$ (que l'on compare avec la préface des Lettres à Serena qui prend le parti des femmes, ou celle de Christianity qui défend le droit à l'éducation de la multitude et des pauvres); et, par ailleurs, il présuppose de leur part une absence de prévention, une disponibilité, qui les rend ouverts à la bonne parole. Assurément, il y a quelque paradoxe à limiter ainsi le public au vu et au su de tous, même des adversaires les plus farouches. Le caractère ésotérique de la confrérie des panthéistes ne va pas jusqu'à imposer un mode de communication lui-même ésotérique. Cependant, l'esprit de controverse a disparu : l'ouvrage se veut serein et dépourvu de querelle. La raison polémique n'est plus.

Mais tous ses traits sont encore présents, et poussés à l'extrême. D'abord, l'ouvrage adopte de façon ouverte le mode de la profession de foi : même l'exposé didactique initial se réduit à la présentation raisonnée (mais non argumentée) d'une sorte de credo (l'auteur abandonne à l'occasion le discours indirect et passe ainsi du récit à la déclaration). Les raisons du panthéisme, de ce dynamisme matérialiste cher à Toland, ne sont pas

46. Pantheisticon of the Form of Celebrating the Socratic-Society (cite par la suite comme Pantheisticon), Londres, Sam. Paterson, 1751, "To the Reader ", p. I-II.

47. Pantheisticon, p. II. 
présentées : il suffit de dire que la plupart de ces sociétés philosophiques secrètes, de l'Antiquité aux Temps modernes, ont épousé cette doctrine, et de mettre au service de celle-ci la bonne foi du philosophe : «For be it from me that I should assert anything that is not proved by experience and reason ${ }^{48}$. Ensuite, que ce soit dans les glissements du discours, dans la répétition de la doctrine sous la forme d'un rituel verbal, touchant les principes du monde, Dieu et l'homme moral, ou encore dans le choix des longues citations de Cicéron ou de Sénèque, tout est confié à la valeur existentielle du canon présenté : le panthéisme est d'abord certitude tranquille, sérénité de l'âme face au préjugé et à la superstition, et absence de crainte envers la mort. Enfin, le modèle proposé est celui d'une confrérie de philosophes qui, lorsqu'ils sont réunis autour d'une table confortable, mais modeste, concourent librement dans la même unanimité.

Ainsi est brossé l'idéal auquel se porte la raison polémique : Épicure plutôt que Socrate. La recherche d'une valeur de vie plutôt que l'examen de la vérité, même si la seconde est le moyen de la première. Aussi longtemps que l'esprit de sérénité n'a pas prévalu, il faut employer l'économie de la polémique pour combattre l'intolérance qui accompagne ordinairement la subordination de la vérité aux valeurs de vie. Quant à la raison ellemême, elle n'est point raisonnement, mais un mode d'assertion et d'exposition. À cet égard, Toland n'est pas étranger à son siècle.

Michel MalHerbe (mai 1994).

48. Pantheisticon, p. 44. 